\title{
Effect of Educational Program for Patient's With Congestive Heart Failure on Their Health Status
}

Prof. Sanaa Mohammed Alaa El-Din; Prof. Ahmed El-Hawary, Assist Prof. Eman Saleh Shahin, Sherin Ebrahim El-Tahry

Prof of Medical Surgical Nursing Department - Faculty of Nursing - Alexandria

University; Prof and Head of Cardiology Department - Suez Canal University Faculty of Medicine; Assist Prof. of Medical Surgical Nursing - Faculty of Nursing Port Said University

\begin{abstract}
Background : The aim of this study is to assess the patients knowledge and evaluate the effect of an educational program on patient outcome as an indicator of health status.Subject and Methods: A quasi-experimental design was used to assess the patient knowledge regarding congestive heart failure and evaluate the effect of implementing an educational program on patient outcomes . The setting of the study was conducted in the cardiac care units in three selected governmental hospitals in Port Said city , El-Nasr Hospital, General Port Said Hospital and Al-Tadamon Health Insurance Hospital (The total sample size was 108 patients).Two tools was developed and used by the researcher for data collection. Tool: Congestive heart failure patients Questionnaire is divided into two parts. Part (1): This part included knowledge assessment regarding congestive heart failure and clinical data questionnaire as sociodemographic data and patient history and clinical data. Part (2): This part included educational program for patients with congestive heart failure. Results: The results revealed that there was a significant improvement in the patient's knowledge about definition, types, incidence, signs and symptom, patient belief about congestive heart failure, psych statues, in the first evaluation (after 1 month) and second evaluation (after 3 months).Conclusion: Educational program has a significant improvement on the patient's knowledge regarding congestive heart failure.
\end{abstract}

Key Words: Congestive heart failure, educational program and health status. 


\section{INTRODUCTION}

Congestive heart failure ( $\mathrm{CHF}$ ) occurs when the heart muscle is not able to pump enough blood to satisfy the metabolic needs of the tissues. Additionally, heart failure may be due to a primary disease of the heart muscle which is called cardiomyopathy or secondary to disease which affects the heart muscle such as hypertension, coronary heart disease, or various diseases of the heart valves (Amorim et al., 2013; Katz et al., 2015). $\mathrm{CHF}$ is often associated with disability and is characterized by left ventricular dysfunction, reduced exercise tolerance, diminished quality of life, and shortened life expectancy (McMurray et al., 2012). As indicated by Zill et al. (2011) a total of $50 \%$ of all patients with CHF die within 5 years of diagnosis.

Congestive heart failure is a complex clinical syndrome in which the patient complains of shortness of breath, fatigue and abnormal heart function. Heart failure is not a diagnosis but it is produced by any disorder that places the heart under a chronically increased volume or pressure load that produces primary damage or an increased metabolic demand on the myocardium (Pinkerman et al., 2013).

Furthermore, congestive heart failure is a significant health problem for men and women. It is characterized by marked functional limitation and poor quality of life, so the patients find themselves in a new life situation in their environment, their family, work and society. They usually face many problems such as loss of some body functions, relationships with others, altered sexuality and change in life style (Walker et al., 2014).

Moser et al. (2012) mentioned that patients with congestive heart failure need to have information regarding their own self-care. This information should include all aspects of their illness and its treatment. Moreover, Weber \& Kelley (2014), indicated that global information needs of patient with congestive heart failure help patient achieve high level of wellness. Moreover, the information that patients need to receive should be related to symptoms, medications, weight monitoring, diet, oxygen therapy, activity and stress management.....etc.

Patient with congestive heart failure should be prepared for transition of care from hospital to home through discharge planning. An effective discharge planning process could be described as the construction for implementation of a planned program of continuing care, which meets patients' needs after discharge from hospital (Urden et al., 2014; Jensen, 2015). The practice nurse plays an important role for patients with CHF. Which assesses patients' information regarding his/her disease to modify treatment and guide the patients and their families to facilitate adaptations with heart failure (Williams \& Hopper, 2015). 


\section{AIM OF STUDY:}

The aim of this study is to assess the patients knowledge and evaluate the effect of an educational program on patient outcome as an indicator of health status.

\section{SUBJECT AND METHODS:}

A quasi-experimental design was used to assess the patient knowledge regarding congestive heart failure and evaluate the effect of educational program for patient with congestive heart failure .The subjects of the study was conducted in the cardiac care units in three selected governmental hospitals in Port Said city, El-Nasr Hospital, General Port Said Hospital and Al-Tadamon Health Insurance Hospital (The total sample size was 108 patients). Conceptual definition: Health status defend as a holistic concept that is determined by more than the presence or absence of any disease. It is often summarized by life expectancy or self-assessed health status, and more broadly includes measures of functioning, physical illness, and mental wellbeing (Australian Institute of Health and Welfare ,2016).

Both study and control groups filled out the questionnaires through structured interview that consist of three assessment before implement program as a pre -test and immediate at the end of the program as post 1 then after three month as follow up test or post 2. then the educational program were conducted only for the study group according to their disease stage, level of education and their free time for most patient the teaching sessions were scheduled in the afternoon that consist of four session. Each session was done every week for one month and lasted for 30-45 minutes depending on certain circumstances such as patients level or depth of discussion.

\section{Inclusion Criteria:}

1. Patients above 40 years of age both sex males and females.

2. Patients with a confirmed diagnosis of congestive heart failure for at least one year.

\section{Exclusion Criteria:}

- Patient inability to communicate due to poor general conditions and deafness hearing alteration.

\section{Tools of Data Collection:}

\section{Part (I) Knowledge Assessment Regarding Congestive Heart Failure and Clinical Data Questionnaire:}

This questionnaire was developed by the researcher after a thorough review of literature, this tool is divided into two main parts.

- Socio-demographic data: such as age, gender, marital status, level of education, job type, caregiver, number of children, family members living with the patient, number of rooms, crowding index, monthly income sufficient for costs of treatment.

- Patient history and clinical data: such as types, diagnosis of CHF, onset of illness, time since start (years), concomitant disease, number of hospitals admission in the last year, signs and symptoms as, edema, complications that occurred , smoking, 
exercise, laboratory investigations, vital signs, control of weight, diet, medication, and sexual relation, (Abu Donia, 1977).

\section{A pilot study:}

A pilot study was carried out on $10 \%$ of the patients. The main purpose of the pilot study was to test the clarity, feasibility of the tools and whether it was understandable, and to determine the time needed to fill the tool. The tool was handed to participants to fill it in and collected by the researcher.

\section{Educational Program:}

The health education program was designed based on; several literature review and the findings of the baseline assessment of CHF patients' knowledge(Helen \& Sharon, 2008; Lakdizaji et al., 2013; Noghabi et al., 2013; Pinkerman et al., 2013 ). The general objectives distributed on four sessions with each session having a set of specific objectives:

- The first session's aim was to: inform CHF patients about the anatomy and physiology of the heart.

- The second session's aim was to: definition of CHF ,risk factors, signs and symptoms .

- The third session's aim was to: control of possible complications,

- The fourth session's aim was to: cope with stress management and medical checkup or follow up.

\section{Field work:}

The program contents and the choice of teaching methods were based on level of education and needs whether individually or in groups. An educational booklet, brochures and videos on copy-right disk $\mathrm{CD}$ were prepared by the researcher to present information for patients in a simple way using simple language and illustrative pictures. It distributed to all patients at the proper time.

Field study was conducted from the beginning of June (2013) to the end of August (2014). Reliability was performed for the tool where Cronbach alpha equals 0.751 .

Conceptual definition: Health statuses defend as a holistic concept that is determined by more than the presence or absence of any disease. It is often summarized by life expectancy or self-assessed health status, and more broadly includes measures of functioning, physical illness, and mental wellbeing (Australian Institute of Health and Welfare, 2016). 


\section{RESULTS:}

Table (1): shows the distribution of socio-demographic characteristics of both study and control groups. None of the characteristics showed significant difference between both groups except type of work practiced by both groups $(p=0.001)$, gender $(p=0.053)$ and carer of family members $(\mathrm{P}=0.029)$.

Table (2): shows the Comparison between study and control groups as regards socioeconomic conditions. Differences in socio-economic conditions of both groups were statistically significantly between both groups. The floor of housing in about half of the study group was at the ground level $(40.7 \%)$ compared to the majority of the control group (79.6\%) who lived in the second floor $(\mathrm{P}=0.001)$. Healthy home was available for half of the study group (50\%) compared to the majority of the control group (85.2\%); $(\mathrm{P}=0.001)$. Monthly income was significantly lower among the control group $(\mathrm{P}=0.001)$. The majority of the control group (85.2\%) had income less than $300 \mathrm{LE}$ compared to $64.8 \%$ of the study group who had income between 301 and 500 LE. Thus the majority of the control group (87\%) had insufficient income compared to $(51.9 \%)$ of the study group $(\mathrm{P}=0.001)$. Health insurance was offered for $(61.1 \%)$ of the study group compared to only $(14.8 \%)$ of the control group. The differences were statistically significant $(\mathrm{P}=0.001)$.

Table (3): reveals the distribution of knowledge about history of the disease among both studied patient and control groups. Knowledge about diagnosis was more encountered among the control group as compared to the study group $(\mathrm{P}=0.001)$. Knowledge about heart failure was more evident among study group as compared to the control group $(\mathrm{P}=0.001)$. Moreover, causes of heart failure were known to $(37 \%)$ of the study group compared to $(14.8 \%)$ of the control group $(\mathrm{P}=0.008)$.

Table (4): shows the medical history of diseases among the studied patient and control groups. All studied and control group patient suffered from hypertension. The majority of the control group suffered from myocardial infarction (77.8\%) compared to (46.3\%) among patient followed by angina pectoris (75.9\%, 37\% respectively) and severe anemia $(66.7 \%, 20.4 \%$ respectively). All these differences were statistically significant.

Regarding onset of disease, the majority of studied- patient, had gradual onset of disease compared to all controls. The difference was statistically significant $(p=0.003)$. The mean number of hospital admissions of both studied and control patient did not show significant difference $(6.1 \pm 5.62,5.9 \pm 5.19$ respectively), $(\mathrm{P}=0.85)$. Cause for admission was almost heart failure combined with other causes among studied patient $(75.9 \%)$ and controls $(66.7 \%)$. The difference was statistically significant $(\mathrm{p}=0.001)$.

Considering knowledge about avoiding symptoms, it was only available among $22.2 \%$ of studied patient and $13 \%$ of controls. Nearly similar percentages were found among those who did not have the knowledge $(31.5 \%, 37 \%$ respectively). 
Table (5): shows significant differences between the studied and control group regarding patients' problems as chest tightness at night, upstairs tachypnea, need for rest during physical activity and need for rest at a specific position. Sleep problems were more encountered among the control group (98.1\%) as compared to the study group (79.6\%). Furthermore, the control group reported shorter durations of sleep. Differences were statistically significant $(\mathrm{P}=0.001$ respectively).

Changing pattern of sleep was equal among both groups (85.2\%). The change of sleep encountered was in the form of decreased sleep among (82.6\%) of study and (97.8\%) of control group. Regarding effect of the disease on sexual life, it was revealed that no significant differences were found between both study and control groups as more than one third of each group reported that there was full interference on sexual life $(35.2 \%$ and $44.4 \%$ respectively).

Table (6): shows the effect of the educational program on emotional state among the study and control group. It was revealed that anyone told about heart failure was not significant in the both group at all times of the evaluation. Moreover, the study group subjects had significantly lower percentage after $1^{\text {st }}$ evaluation for increase anxiety by the disease, less affection on behavior and vision of life as compared to the control group at all times of evaluation.

Regarding the presence of a positive effect for the disease on life of the patient, it was found that it was less reported among the study group before the program while they showed increase in such beliefs after the program as compared to the control group.

No significant difference was found between study and controls regarding change of their life pattern completely after the illness as the majority of both groups confirmed such change in life pattern.

A significantly higher percentage of control group felt the diseases leads to social loneliness more than the study group $(\mathrm{P}=0.001)$. The study group who felt the diseases leads to social loneliness decreased after $1^{\text {st }}$ evaluation of the program.

The majority of the control group felt anxious when they knew the diagnosis compared to significant lower percentage among the study group before and immediately after the program. Meanwhile, at $2^{\text {nd }}$ evaluation after the program, it was found that a significantly higher percentage of the study group reported that they had decreased anxiety $(\mathrm{P}=0.013)$.

No statistically significant difference was found between study and control group, regarding belief afraid from disease complications ( $\mathrm{p}=495)$. The study group had decreased percentage from $(96.3 \%)$ before program to $(94.4 \%)$, after $1^{\text {st }}$ evaluation, again it increased to $(96.3 \%)$ after $2^{\text {nd }}$ evaluation. While in the control group did not change before or after the $1^{\text {st }}$ and $2^{\text {nd }}$ evaluation (100\%). 
Regarding the ability to concentrate and remember things, it was significantly higher among the control group as compared to the study group at all times. Again, a higher percentage of the study group was satisfied about their life, which increased after $1^{\text {st }}$ evaluation of the program as compared to the control group.

Table (7): shows the effect of the educational program on opinion among study and control groups. Regarding patient belief about heart failure, $44.4 \%$ of the study group thought that heart failure disables patient completely compared to none of the control group before the program. After the program, more than half of the study group $(57.4 \%$, 74.1 respectively) thought that heart failure is inability of heart to pump enough blood compared to $(25.9 \%)$ which is statistically significant $(\mathrm{P}=0.001)$.

Regarding the most important factor affecting the disease, the majority of the study group thought that nothing could affect the disease (37\%) or all presented factors except weakness of pulse rate $(35.2 \%)$ before the program. Meanwhile, half of the control group (51.9\%) thought that the most important factor is psychic rest. After the program, nearly all the study group (92.6\%) thought that all factors could affect the disease except weakness of pulse compared to $42.6 \%$ among the control group. On second evaluation after the program, $83.3 \%$ of the study group thought that all factors would affect the disease compared to $40.7 \%$ of the control group. All differences were statistically significant $(\mathrm{P}=0.001)$. The most difficult disease as being reported by the study group before the program was heart disease $(68.5 \%)$ compared to $46.3 \%$ among the control group.

Similarly after the program, the highest percentage of study group (81.5\% and $75.9 \%)$ believed that heart disease is the most difficult compared to significantly lower percentage among the control group (48.1\%). All differences were statistically significant $(\mathrm{P}=0.001)$. 
Table (1): Distribution of socio-demographic characteristics of the study and control group

\begin{tabular}{|c|c|c|c|c|}
\hline $\begin{array}{l}\text { Socio-demographic } \\
\text { characteristics }\end{array}$ & $\begin{array}{l}\text { Study } \\
\text { group } \\
(n=54)\end{array}$ & $\begin{array}{c}\text { Control } \\
\text { group } \\
(n=54)\end{array}$ & $\begin{array}{c}\text { Total } \\
(\mathrm{n}=108)\end{array}$ & $P$ value \\
\hline \multicolumn{5}{|l|}{ Gender } \\
\hline Male & $25(46.3 \%)$ & $35(64.8 \%)$ & $60(55.6 \%)$ & \multirow[t]{2}{*}{$0.053^{*}$} \\
\hline Female & $29(53.7 \%)$ & $1935.2 \%$ & $48(44.4 \%)$ & \\
\hline \multicolumn{4}{|l|}{ Age (years) } & \multirow{2}{*}{0.36} \\
\hline Mean \pm SD & $57.62 \pm 7.03$ & $56.24 \pm 8.58$ & $56.93 \pm 7.84$ & \\
\hline \multicolumn{4}{|l|}{ Social state } & \multirow{5}{*}{0.29} \\
\hline Single & $4(7.4 \%)$ & $6(11.1 \%)$ & $10(9.3 \%)$ & \\
\hline Married & $38(70.4 \%)$ & $33(61.1 \%)$ & $71(65.7 \%)$ & \\
\hline Widow & $12(22.2 \%$ & $12(22.2 \%)$ & $24(22.2 \%)$ & \\
\hline Divorced & $0(0.0 \%)$ & $3(5.6 \%)$ & $3(2.8 \%)$ & \\
\hline \multicolumn{4}{|l|}{ Educational level } & \multirow{5}{*}{0.065} \\
\hline Illiterate & $13(24.1 \%)$ & $19(35.2 \%)$ & $32(29.6 \%)$ & \\
\hline Read and write & $22(40.7 \%)$ & $25(46.3 \%)$ & $47(43.5 \%)$ & \\
\hline Prep school & $2(3.7 \%)$ & $4(7.4 \%)$ & $6(5.6 \%)$ & \\
\hline Secondary or above & $17(31.5 \%)$ & $6(11.1 \%)$ & $23(21.3 \%)$ & \\
\hline \multicolumn{4}{|l|}{ Employment } & \multirow{3}{*}{0.11} \\
\hline No & $23(42.6 \%)$ & $15(27.8 \%)$ & $38(35.2 \%)$ & \\
\hline Yes & $31(57.4 \%)$ & $39(72.2 \%)$ & $70(64.8 \%)$ & \\
\hline Type of work & $(\mathrm{n}=31)$ & $(n=39)$ & $(n=70)$ & \multirow{3}{*}{$0.001 *$} \\
\hline Physical & $21(67.7 \%)$ & $38(97.4 \%)$ & $59(84.3 \%)$ & \\
\hline Mental & $10(32.3 \%)$ & $1(2.6 \%)$ & $11(15.7 \%)$ & \\
\hline \multicolumn{4}{|l|}{ Carer } & \multirow{5}{*}{$0.029 *$} \\
\hline Family & $32(59.3 \%)$ & $39(72.2 \%)$ & $71(65.7 \%)$ & \\
\hline One of sons & $13(24.1 \%)$ & $13(24.1 \%)$ & $26(24.1 \%)$ & \\
\hline Brother & $1(1.9 \%)$ & $2(3.7 \%)$ & $3(2.8 \%)$ & \\
\hline No one & $8(14.8 \%)$ & $0(0.0 \%)$ & $8(7.4 \%)$ & \\
\hline \multicolumn{4}{|l|}{ Residence } & \multirow{3}{*}{0.3} \\
\hline Special & $43(79.6 \%)$ & $47(87.0 \%)$ & $90(83.3 \%)$ & \\
\hline with others & $11(20.4 \%)$ & $7(13.0 \%)$ & $18(16.7 \%)$ & \\
\hline
\end{tabular}

*significant level considered- $P \leq 0.05$ 
Table (2): Comparison between study and control groups as regards socio-economic conditions

\begin{tabular}{|c|c|c|c|c|}
\hline $\begin{array}{l}\text { Socio-economic } \\
\text { conditions }\end{array}$ & $\begin{array}{l}\text { Study group } \\
\quad(n=54)\end{array}$ & $\begin{array}{l}\text { Control } \\
\text { group } \\
(n=54)\end{array}$ & $\begin{array}{c}\text { Total } \\
(n=108)\end{array}$ & $P$ value \\
\hline $\begin{array}{l}\text { Floor of housing } \\
\text { At the ground } \\
\text { First } \\
\text { Second } \\
\text { Third } \\
\text { Others }\end{array}$ & $\begin{array}{c}22(40.7 \%) \\
6(11.1 \%) \\
17(31.5 \%) \\
5(9.3 \%) \\
4(7.4 \%)\end{array}$ & $\begin{array}{c}7(13.0 \%) \\
2(3.7 \%) \\
43(79.6 \%) \\
0(0.0 \%) \\
2(3.7 \%)\end{array}$ & $\begin{aligned} 29 & (26.9 \%) \\
8 & (7.4 \%) \\
60 & (55.6 \%) \\
5 & (4.6 \%) \\
6 & (5.6 \%)\end{aligned}$ & $0.001 *$ \\
\hline $\begin{array}{l}\text { Elevator } \\
\text { Present } \\
\text { Absent }\end{array}$ & $\begin{array}{c}4(7.4 \%) \\
50(92.6 \%)\end{array}$ & $\begin{array}{c}2(3.7 \%) \\
52(96.3 \%)\end{array}$ & $\begin{array}{c}6(5.6 \%) \\
102(94.4 \%)\end{array}$ & 0.4 \\
\hline $\begin{array}{l}\text { Healthy home } \\
\text { Yes } \\
\text { No }\end{array}$ & $\begin{array}{l}27(50.0 \%) \\
27(50.0 \%)\end{array}$ & $\begin{array}{c}46(85.2 \%) \\
8(14.8 \%)\end{array}$ & $\begin{array}{l}73(67.6 \%) \\
35(32.4 \%)\end{array}$ & $0.001 *$ \\
\hline $\begin{array}{l}\text { Monthly income } \\
\text { Less than } 300 \\
\text { From } 301-500 \\
\text { More than } 500\end{array}$ & $\begin{array}{c}17(31.5 \%) \\
35(64.8 \%) \\
2(3.7 \%)\end{array}$ & $\begin{array}{c}46(85.2 \%) \\
8(14.8 \%) \\
0(0.0 \%)\end{array}$ & $\begin{array}{c}63(58.3 \%) \\
43(39.8 \%) \\
2(1.9 \%)\end{array}$ & $0.001 *$ \\
\hline $\begin{array}{l}\text { Sufficiency of income } \\
\text { Yes } \\
\text { No } \\
\text { Somewhat }\end{array}$ & $\begin{array}{l}13(24.1 \%) \\
28(51.9 \%) \\
13(24.1 \%)\end{array}$ & $\begin{array}{c}7(13.0 \%) \\
47(87.0 \%) \\
0(0.0 \%)\end{array}$ & $\begin{array}{l}20(18.5 \%) \\
75(69.4 \%) \\
13(12.0 \%)\end{array}$ & $0.001 *$ \\
\hline $\begin{array}{l}\text { Health insurance } \\
\text { Yes } \\
\text { No }\end{array}$ & $\begin{array}{l}33(61.1 \%) \\
21(38.9 \%)\end{array}$ & $\begin{array}{l}8(14.8 \%) \\
46(85.2 \%)\end{array}$ & $\begin{array}{l}41(38.0 \%) \\
67(62.0 \%)\end{array}$ & $0.001 *$ \\
\hline
\end{tabular}

*significant level considered- $P \leq 0.05$

Table (3): Knowledge about history of the disease in study and control groups

\begin{tabular}{|c|c|c|c|c|c|}
\hline \multicolumn{2}{|c|}{$\begin{array}{l}\text { Knowledge about disease } \\
\text { history }\end{array}$} & $\begin{array}{l}\text { Study } \\
\text { group } \\
(\mathrm{n}=54)\end{array}$ & $\begin{array}{c}\text { Control group } \\
(\mathbf{n}=54)\end{array}$ & $\begin{array}{c}\text { Total } \\
(n=108)\end{array}$ & $P$ value \\
\hline $\begin{array}{l}\text { Know diagnosis } \\
\text { of the disease }\end{array}$ & $\begin{array}{l}\text { Yes } \\
\text { No }\end{array}$ & $\begin{array}{l}40(74.1 \%) \\
14(25.9 \%)\end{array}$ & $\begin{array}{c}52(96.3 \%) \\
2(3.7 \%)\end{array}$ & $\begin{array}{l}92(85.2 \%) \\
16(14.8 \%)\end{array}$ & $0.001 *$ \\
\hline Know heart failure & $\begin{array}{l}\text { Yes } \\
\text { No }\end{array}$ & $\begin{array}{l}36(66.7 \%) \\
18(33.3 \%)\end{array}$ & $\begin{array}{l}16(29.6 \%) \\
38(70.4 \%)\end{array}$ & $\begin{array}{l}52(48.1 \%) \\
56(51.9 \%)\end{array}$ & $0.001 *$ \\
\hline $\begin{array}{l}\text { Cause of heart } \\
\text { failure }\end{array}$ & $\begin{array}{l}\text { Yes } \\
\text { No }\end{array}$ & $\begin{array}{l}20(37.0 \%) \\
34(63.0 \%)\end{array}$ & $\begin{array}{c}8(14.8 \%) \\
46(85.2 \%)\end{array}$ & $\begin{array}{l}28(25.9 \%) \\
80(74.1 \%)\end{array}$ & $0.008 *$ \\
\hline Type of heart failure & $\begin{array}{l}\text { Yes } \\
\text { No }\end{array}$ & $\begin{array}{l}10(18.5 \%) \\
44(81.5 \%)\end{array}$ & $\begin{array}{c}1(1.9 \%) \\
53(98.1 \%)\end{array}$ & $\begin{array}{l}11(10.2 \%) \\
97(89.8 \%)\end{array}$ & $0.004 *$ \\
\hline
\end{tabular}

*significant level considered- $\quad P \leq 0.05$ 
Table (4): Medical history of the disease among the study and control groups

\begin{tabular}{|c|c|c|c|c|c|}
\hline \multicolumn{2}{|c|}{ Medical history } & $\begin{array}{l}\text { Study } \\
\text { group } \\
(\mathrm{n}=54)\end{array}$ & $\begin{array}{l}\text { Control } \\
\text { group } \\
(n=54)\end{array}$ & $\begin{array}{c}\text { Total } \\
(\mathbf{n}=\mathbf{1 0 8})\end{array}$ & $\begin{array}{c}\mathbf{P} \\
\text { value }\end{array}$ \\
\hline \multirow{8}{*}{$\begin{array}{l}\text { Previous } \\
\text { disease }\end{array}$} & Hypertension & $54(100 \%)$ & $54(100 \%)$ & $108(100 \%)$ & - \\
\hline & MI & $25(46.3 \%)$ & $42(77.8 \%)$ & $67(62.0 \%)$ & $0.001 *$ \\
\hline & Rheumatic heart & $7(13.0 \%)$ & $2(3.7 \%)$ & $9(8.3 \%)$ & 0.08 \\
\hline & Angina pectoris & $20(37.0 \%)$ & $41(75.9 \%)$ & $61(56.5 \%)$ & $0.001 *$ \\
\hline & Valvular stenosis & $7(13.0 \%)$ & $0(0.0 \%)$ & $7(6.5 \%)$ & $0.019 *$ \\
\hline & Severe anemia & $11(20.4 \%)$ & $36(66.7 \%)$ & $47(43.5 \%)$ & $0.001 *$ \\
\hline & Hyperthyroidism & $4(7.4 \%)$ & $7(13.0 \%)$ & $11(10.2 \%)$ & 0.34 \\
\hline & Bronchial asthma & $13(24.1 \%)$ & $5(9.3 \%)$ & $18(16.7 \%)$ & $0.03 *$ \\
\hline \multirow[t]{2}{*}{ Diagnosis } & Sudden & $8(14.8 \%)$ & $0(0.0 \%)$ & $8(7.4 \%)$ & \multirow{2}{*}{$0.003 *$} \\
\hline & Gradual & $46(85.2 \%)$ & $54(100.0 \%)$ & $100(92.6 \%)$ & \\
\hline \multirow[t]{2}{*}{ Duration } & One year & $2(3.7 \%)$ & $0(0.0 \%)$ & $2(1.9 \%)$ & \multirow{2}{*}{0.15} \\
\hline & More than one year & $52(96.3 \%)$ & $54(100.0 \%)$ & $106(98.1 \%)$ & \\
\hline \multirow[t]{4}{*}{ Other diseases } & Diabetes & $2(3.7 \%)$ & $0(0.0 \%)$ & $2(1.9 \%)$ & \multirow{4}{*}{$0.021 *$} \\
\hline & Hypertension & $5(9.3 \%)$ & $0(0.0 \%)$ & $5(4.6 \%)$ & \\
\hline & DM and HTN & $46(85.2 \%)$ & $49(90.7 \%)$ & $95(88.0 \%)$ & \\
\hline & Others & $1(1.9 \%)$ & $5(9.3 \%)$ & $6(5.6 \%)$ & \\
\hline \multicolumn{2}{|c|}{ Admission in last year $($ mean \pm SD) } & $6.09 \pm 5.62$ & $5.90 \pm 5.19$ & $6.0 \pm 5.38$ & 0.85 \\
\hline \multirow{5}{*}{$\begin{array}{l}\text { Duration of } \\
\text { admission }\end{array}$} & One week & $22(40.7 \%)$ & $7(13.0 \%)$ & $29(26.9 \%)$ & \multirow{5}{*}{$0.001^{*}$} \\
\hline & Two weeks & $14(25.9 \%)$ & $39(72.2 \%)$ & $53(49.1 \%)$ & \\
\hline & Three weeks & $7(13.0 \%)$ & $1(1.9 \%)$ & $8(7.4 \%)$ & \\
\hline & One month & $7(13.0 \%)$ & $5(9.3 \%)$ & $12(11.1 \%)$ & \\
\hline & $\begin{array}{l}\text { Others (more than one } \\
\text { month) }\end{array}$ & $4(7.4 \%)$ & $2(3.7 \%)$ & $6(5.6 \%)$ & \\
\hline \multirow{3}{*}{$\begin{array}{l}\text { Cause of } \\
\text { admission }\end{array}$} & $\mathrm{HF}$ & $12(22.2 \%)$ & $0(0.0 \%)$ & $12(11.1 \%)$ & \multirow{3}{*}{$0.001 *$} \\
\hline & Others & $1(1.9 \%)$ & $18(33.3 \%)$ & $19(17.6 \%)$ & \\
\hline & HF and others & $41(75.9 \%)$ & $36(66.7 \%)$ & $77(71.3 \%)$ & \\
\hline \multirow[t]{4}{*}{ Visit doctor } & At the same week & $31(57.4 \%)$ & $29(53.7 \%)$ & $60(55.6 \%)$ & \multirow{4}{*}{0.10} \\
\hline & At the same month & $18(33.3 \%)$ & $25(46.3 \%)$ & $43(39.8 \%)$ & \\
\hline & After 3 months & $3(5.6 \%)$ & $0(0.0 \%)$ & $3(2.8 \%)$ & \\
\hline & After 6 months & $2(3.7 \%)$ & $0(0.0 \%)$ & $2(1.9 \%)$ & \\
\hline \multirow{3}{*}{$\begin{array}{l}\text { Know how to } \\
\text { avoid } \\
\text { symptoms }\end{array}$} & Yes & $12(22.2 \%)$ & $7(13.0 \%)$ & $19(17.6 \%)$ & \multirow{3}{*}{0.44} \\
\hline & No & $17(31.5 \%)$ & $20(37.0 \%)$ & $37(34.3 \%)$ & \\
\hline & Somewhat & $25(46.3 \%)$ & $27(50.0 \%)$ & $52(48.1 \%)$ & \\
\hline
\end{tabular}

N.B. No cases reported previous congenital heart disease or chronic chest disease *significant level considered- $\mathbf{P} \leq \mathbf{0 . 0 5}$ 
Table (5): Comparison between study and control groups as regards data of patient's problems

\begin{tabular}{|c|c|c|c|c|c|}
\hline \multicolumn{2}{|c|}{ Patients needs } & $\begin{array}{l}\text { Study } \\
\text { group } \\
(n=54)\end{array}$ & $\begin{array}{c}\text { Control } \\
\text { group } \\
(n=54)\end{array}$ & $\begin{array}{c}\text { Total } \\
(\mathbf{n}=\mathbf{1 0 8})\end{array}$ & $P$ value \\
\hline \multirow{2}{*}{$\begin{array}{l}\text { Chest tightness at } \\
\text { effort }\end{array}$} & Yes & $39(72.2 \%)$ & $35(64.8 \%)$ & $74(68.5 \%)$ & \multirow{2}{*}{0.407} \\
\hline & No & $15(27.8 \%)$ & $19(35.2 \%)$ & $34(31.5 \%)$ & \\
\hline \multirow[t]{2}{*}{ Anxiety on leaning } & Yes & $42(77.8 \%)$ & $35(64.8 \%)$ & $77(71.3 \%)$ & \multirow{2}{*}{0.136} \\
\hline & No & $12(22.2 \%)$ & $19(35.2 \%)$ & $31(28.7 \%)$ & \\
\hline \multirow{2}{*}{$\begin{array}{l}\text { Chest tightness at } \\
\text { night }\end{array}$} & Yes & $50(92.6 \%)$ & $36(66.7 \%)$ & $86(79.6 \%)$ & \multirow{2}{*}{$0.001 *$} \\
\hline & No & $4(7.4 \%)$ & $18(33.3 \%)$ & $22(20.4 \%)$ & \\
\hline \multirow[t]{2}{*}{ Upstairs tachypnea } & Yes & $51(94.4 \%)$ & $35(64.8 \%)$ & $86(79.6 \%)$ & \multirow{2}{*}{$0.001 *$} \\
\hline & No & $3(5.6 \%)$ & $19(35.2 \%)$ & $22(20.4 \%)$ & \\
\hline \multirow{2}{*}{$\begin{array}{l}\text { Rest during } \\
\text { physical activity }\end{array}$} & Yes & $50(92.6 \%)$ & $35(64.8 \%)$ & $85(78.2 \%)$ & \multirow{2}{*}{$0.001 *$} \\
\hline & No & $4(7.4 \%)$ & $19(35.2 \%)$ & $23(21.3 \%)$ & \\
\hline \multirow{2}{*}{$\begin{array}{l}\text { Rest at a specific } \\
\text { position }\end{array}$} & Yes & $47(87.0 \%)$ & $8(14.8 \%)$ & $55(50.9 \%)$ & \multirow{2}{*}{$0.001 *$} \\
\hline & No & $7(13.0 \%)$ & $46(85.2 \%)$ & $53(49.1 \%)$ & \\
\hline \multirow[t]{8}{*}{$\begin{array}{l}\text { Complications of } \\
\text { prolonged bed rest }\end{array}$} & $\begin{array}{l}\text { Muscle } \\
\text { weakness }\end{array}$ & $30(55.6 \%)$ & $6(11.1 \%)$ & $36(33.3 \%)$ & \multirow{8}{*}{$\begin{array}{c}0.001^{*} \\
0.001^{*} \\
0.12 \\
0.51 \\
0.001^{*} \\
0.001^{*} \\
0.001^{*} \\
0.19\end{array}$} \\
\hline & Bed sores & $30(55.6 \%)$ & $13(24.1 \%)$ & $43(39.8 \%)$ & \\
\hline & Pneumonia & $4(7.4 \%)$ & $0(0.0 \%)$ & $4(3.7 \%)$ & \\
\hline & DVT & $13(24.1 \%)$ & $16(29.6 \%)$ & $29(26.9 \%)$ & \\
\hline & Arthrodesis & $0(0.0 \%)$ & $39(72.2 \%)$ & $39(36.1 \%)$ & \\
\hline & Constipation & $29(53.7 \%)$ & $3(5.6 \%)$ & $32(29.6 \%)$ & \\
\hline & Urine retention & $17(31.5 \%)$ & $1(1.9 \%)$ & $18(16.7 \%)$ & \\
\hline & Dyspnea & $18(33.3 \%)$ & $12(22.2 \%)$ & $30(27.8 \%)$ & \\
\hline \multirow[t]{2}{*}{ Sleep problems } & Yes & $43(79.6 \%)$ & $53(98.1 \%)$ & $96(88.9 \%)$ & \multirow{2}{*}{$0.002 *$} \\
\hline & No & $11(20.4 \%)$ & $1(1.9 \%)$ & $12(11.1 \%)$ & \\
\hline \multirow[t]{3}{*}{ Duration of sleep } & 6- 8 hours & $14(25.9 \%)$ & $19(35.2 \%)$ & $33(30.6 \%)$ & \multirow{3}{*}{$0.001 *$} \\
\hline & 4- 6 hours & $25(46.3 \%)$ & $7(13.0 \%)$ & $32(29.6 \%)$ & \\
\hline & $\begin{array}{l}\text { Less than } 4 \\
\text { hours }\end{array}$ & $15(27.8 \%)$ & $28(51.9 \%)$ & $43(39.8 \%)$ & \\
\hline \multirow{2}{*}{$\begin{array}{l}\text { Change of sleep } \\
\text { pattern }\end{array}$} & Yes & $46(85.2 \%)$ & $46(85.2 \%)$ & $92(85.2 \%)$ & \multirow{2}{*}{1.0} \\
\hline & No & $22(14.8 \%)$ & $22(14.8 \%)$ & $44(14.8 \%)$ & \\
\hline \multirow[t]{2}{*}{ Type of change } & Increased & $8(17.4 \%)$ & $1(2.2 \%)$ & $9(9.8 \%)$ & \multirow{2}{*}{0.064} \\
\hline & Decreased & $38(82.6 \%)$ & $45(97.8 \%)$ & $83(90.2 \%)$ & \\
\hline \multirow{2}{*}{$\begin{array}{l}\text { Difficulty in start } \\
\text { night sleep }\end{array}$} & Yes & $50(92.6 \%)$ & $54(100 \%)$ & $104(96.3 \%)$ & \multirow{2}{*}{0.118} \\
\hline & No & $4(7.4 \%)$ & $0(0.0 \%)$ & $4(3.7 \%)$ & \\
\hline \multirow[t]{2}{*}{ Getting up at night } & Yes & $40(74.1 \%)$ & $53(98.1 \%)$ & $93(86.1 \%)$ & \multirow{2}{*}{$0.001 *$} \\
\hline & No & $14(25.9 \%)$ & $1(1.9 \%)$ & $15(13.9 \%)$ & \\
\hline Effect of disease & No at all & $3(5.6 \%)$ & $0(0.0 \%)$ & $3(2.8 \%)$ & \\
\hline on sexual life & Somewhat & $18(33.3 \%)$ & $18(33.3 \%)$ & $36(33.3 \%)$ & 0.29 \\
\hline & $\begin{array}{l}\text { Full } \\
\text { interference }\end{array}$ & $19(35.2 \%)$ & $24(44.4 \%)$ & $43(39.8 \%)$ & \\
\hline & Not answered & $14(25.9 \%)$ & $12(22.2 \%)$ & $26(24.1 \%)$ & \\
\hline
\end{tabular}


Table (5) ): Comparison between study and control groups as regards data of patient's problems (cont)

\begin{tabular}{|c|c|c|c|c|c|}
\hline \multicolumn{2}{|c|}{ Patients needs } & $\begin{array}{l}\text { Study } \\
\text { group } \\
(n=54)\end{array}$ & $\begin{array}{c}\text { Control } \\
\text { group } \\
(n=54)\end{array}$ & $\begin{array}{c}\text { Total } \\
(n=108)\end{array}$ & $P$ value \\
\hline \multicolumn{6}{|c|}{$\begin{array}{l}\text { Sings of heart failure during } \\
\text { sexual activity }\end{array}$} \\
\hline \multirow{2}{*}{$\begin{array}{l}\text { Tachycardia or } \\
\text { tachpnea }\end{array}$} & Yes & $40(100 \%)$ & $42(100 \%)$ & $82(100 \%)$ & \multirow{2}{*}{-NA- } \\
\hline & No & $0(0 \%)$ & $0(0 \%)$ & $0(0 \%)$ & \\
\hline \multirow{2}{*}{$\begin{array}{l}\text { Palpitations for } \\
15 \text { minutes }\end{array}$} & Yes & $32(80 \%)$ & $42(100 \%)$ & $74(90.2 \%)$ & \multirow{2}{*}{$0.002 *$} \\
\hline & No & $8(20 \%)$ & $0(0.0 \%)$ & $8(9.8 \%)$ & \\
\hline \multirow[t]{2}{*}{ Chest pain } & Yes & $31(77.5 \%)$ & $42(100 \%)$ & $73(89 \%)$ & \multirow{2}{*}{$0.001 *$} \\
\hline & No & $9(22.5 \%)$ & $0(0.0 \%)$ & $9(11 \%)$ & \\
\hline \multirow{2}{*}{$\begin{array}{l}\text { Difficult } \\
\text { sleeping after }\end{array}$} & Yes & $35(87.5 \%)$ & $42(100 \%)$ & $77(93.9 \%)$ & \multirow{2}{*}{$0.024 *$} \\
\hline & No & $5(12.5 \%)$ & $0(0.0 \%)$ & $5(6.1 \%)$ & \\
\hline \multirow{2}{*}{$\begin{array}{l}\text { Severe fatigue } \\
\text { After it }\end{array}$} & Yes & $36(90 \%)$ & $42(100 \%)$ & $78(95.1 \%)$ & \multirow{2}{*}{0.053} \\
\hline & No & $4(10 \%)$ & $0(0.0 \%)$ & $4(4.9 \%)$ & \\
\hline \multicolumn{2}{|l|}{ Medical progress } & & & & \\
\hline \multirow[t]{2}{*}{ Prognosis } & Satisfied & $9(16.7 \%)$ & $18(33.3 \%)$ & $27(25 \%)$ & \multirow{2}{*}{$0.046^{*}$} \\
\hline & Unsatisfied & $45(83.3 \%)$ & $36(66.7 \%)$ & $81(75 \%)$ & \\
\hline \multirow[t]{2}{*}{$\begin{array}{l}\text { Change in } \\
\text { prognosis }\end{array}$} & Yes & $53(98.1 \%)$ & $54(100 \%)$ & $\begin{array}{c}107 \\
(99.1 \%)\end{array}$ & \multirow[t]{2}{*}{1.0} \\
\hline & No & $1(1.9 \%)$ & $0(0.0 \%)$ & $1(0.9 \%)$ & \\
\hline
\end{tabular}

NA: Not Applicable

*significant level considered- $\quad P \leq 0.05$ 
Table (6): Effect of the educational program on psychic (or emotional) state among study and control groups at three phases: before, at first and second evaluations

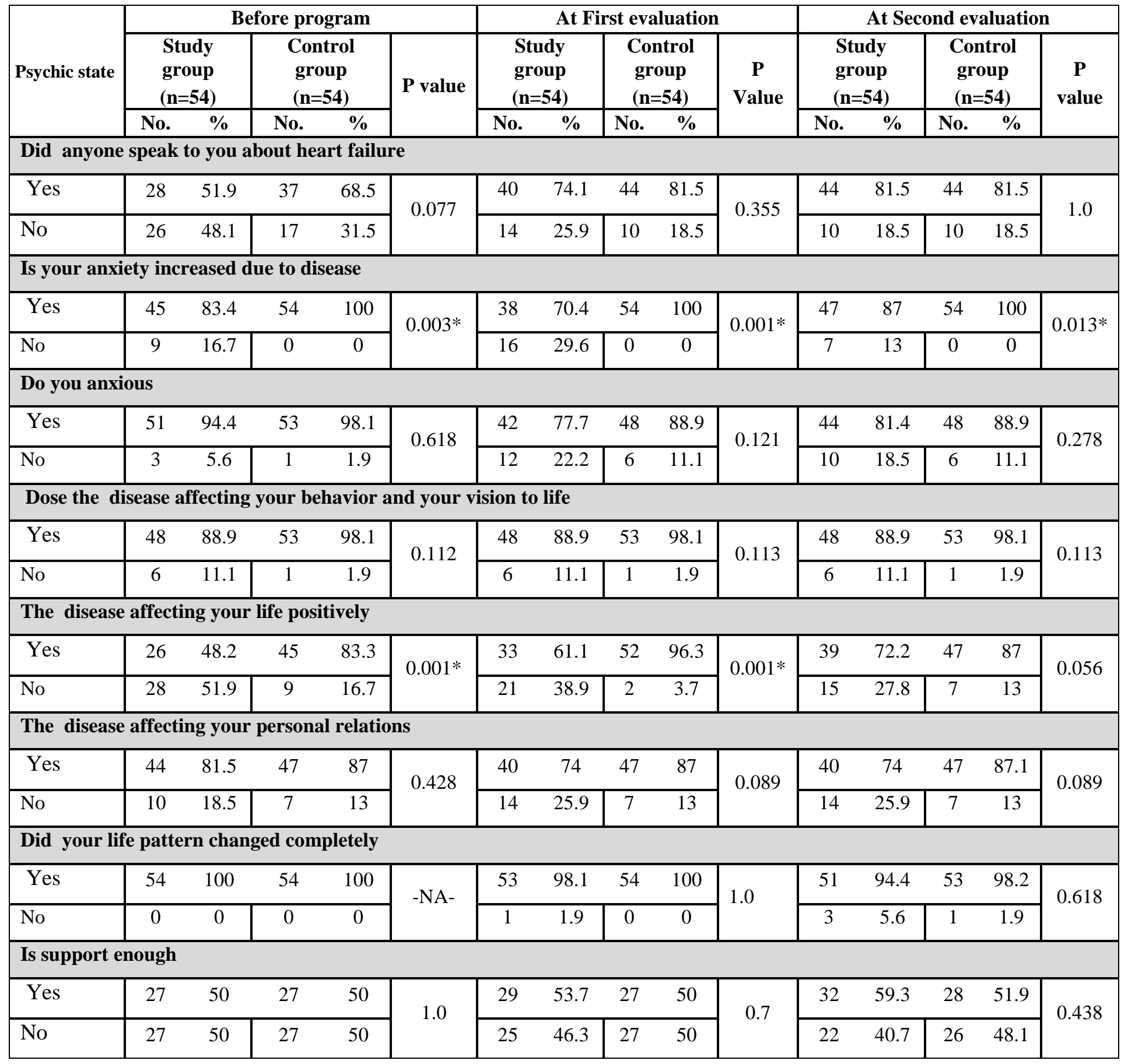


Table (6) (cont): Effect of the educational program on psychic (or emotional) state among study and control groups at three phases: before, at first and second evaluations (cont)

\begin{tabular}{|c|c|c|c|c|c|c|c|c|c|c|c|c|c|c|c|}
\hline \multirow{3}{*}{$\begin{array}{c}\text { Psychic } \\
\text { state }\end{array}$} & \multicolumn{5}{|c|}{ Before program } & \multicolumn{5}{|c|}{ At First evaluation } & \multicolumn{5}{|c|}{ At Second evaluation } \\
\hline & \multicolumn{2}{|c|}{$\begin{array}{l}\text { Study } \\
\text { group } \\
(n=54)\end{array}$} & \multicolumn{2}{|c|}{$\begin{array}{c}\text { Control } \\
\text { group } \\
(n=54)\end{array}$} & \multirow[t]{2}{*}{$\begin{array}{c}\mathbf{P} \\
\text { value }\end{array}$} & \multicolumn{2}{|c|}{$\begin{array}{l}\text { Study } \\
\text { group } \\
(n=54)\end{array}$} & \multicolumn{2}{|c|}{$\begin{array}{c}\text { Control } \\
\text { group } \\
(n=54)\end{array}$} & \multirow[t]{2}{*}{$\begin{array}{c}\mathbf{P} \\
\text { value }\end{array}$} & \multicolumn{2}{|c|}{$\begin{array}{l}\text { Study } \\
\text { group } \\
(n=54)\end{array}$} & \multicolumn{2}{|c|}{$\begin{array}{c}\text { Control } \\
\text { group } \\
(n=54)\end{array}$} & \multirow[t]{2}{*}{$\begin{array}{c}\mathbf{P} \\
\text { value }\end{array}$} \\
\hline & No. & $\%$ & No. & $\%$ & & No. & $\%$ & No. & $\%$ & & No. & $\%$ & No. & $\%$ & \\
\hline \multicolumn{16}{|c|}{ Is the disease leading to social loneliness } \\
\hline Yes & 45 & 83.3 & 54 & 100 & \multirow{2}{*}{$0.003^{*}$} & 37 & 68.5 & 54 & 100 & \multirow{2}{*}{$0.001 *$} & 44 & 81.4 & 54 & 100 & \multirow{2}{*}{$0.001 *$} \\
\hline No & 9 & 16.7 & 0 & 0 & & 17 & 31.5 & 0 & 0 & & 10 & 18.5 & 0 & 0 & \\
\hline \multicolumn{16}{|c|}{ Are you feeling anxious when you know the diagnosis } \\
\hline Yes & 52 & 96.3 & 54 & 100 & \multirow{2}{*}{0.495} & 51 & 94.5 & 54 & 100 & \multirow{2}{*}{0.242} & 47 & 87 & 54 & 100 & \multirow{2}{*}{$0.013^{*}$} \\
\hline No & 2 & 3.7 & 0 & 0 & & 3 & 5.6 & 0 & 0 & & 7 & 13 & 0 & 0 & \\
\hline \multicolumn{16}{|c|}{ Are you afraid from disease complications } \\
\hline Yes & 52 & 96.3 & 54 & 100 & \multirow{2}{*}{0.495} & 51 & 94.4 & 54 & 100 & \multirow{2}{*}{0.242} & 52 & 96.3 & 54 & 100 & \multirow{2}{*}{0.495} \\
\hline No & 2 & 3.7 & 0 & 0 & & 3 & 5.6 & 0 & 0 & & 2 & 3.7 & 0 & 0 & \\
\hline \multicolumn{16}{|c|}{ Is the disease affecting your quality of life } \\
\hline Yes & 52 & 96.3 & 54 & 100 & \multirow{2}{*}{0.495} & 48 & 88.9 & 54 & 100 & \multirow{2}{*}{$0.027 *$} & 52 & 96.3 & 54 & 100 & \\
\hline No & 2 & 3.7 & 0 & 0 & & 6 & 11.1 & 0 & 0 & & 2 & 3.7 & 0 & 0 & ניד. \\
\hline Are you & to & centr & anc & nemb & things & & & & & & & & & & \\
\hline Yes & 43 & 79.6 & 54 & 100 & & 51 & 94.4 & 54 & 100 & & 47 & 87 & 54 & 100 & \\
\hline No & 11 & 20.4 & 0 & 0 & & 3 & 5.6 & 0 & 0 & & 7 & 13 & 0 & 0 & \\
\hline Are you s & fiod & ith yc & r life & & & & & & & & & & & & \\
\hline Yes & 32 & 59.3 & 41 & 75.9 & & & 92.6 & 44 & 81.5 & & 46 & 85.2 & 44 & 81.5 & \\
\hline No & 22 & 40.7 & 13 & 24.1 & & 4 & 7.4 & 10 & 18.5 & & 8 & 14.8 & 10 & 18.5 & \\
\hline Do religi & & ies su & ort & regar & ig your & isease & & & & & & & & & \\
\hline Yes & 53 & 98.1 & 54 & 100 & & 54 & 100 & 54 & 100 & & 54 & 100 & 54 & 100 & \\
\hline No & 1 & 1.9 & 0 & 0 & 1.0 & 0 & 0 & 0 & 0 & 171 & 0 & 0 & 0 & 0 & 171 \\
\hline Do you $f$ & . & an a & 101 & life & & & & & & & & & & & \\
\hline Yes & 38 & 70.4 & 31 & 57.4 & & & 98.1 & 33 & 61.1 & & 52 & 96.3 & 33 & 61.1 & \\
\hline No & 16 & 29.6 & 23 & 42.6 & & 1 & 1.9 & 21 & 38.9 & & 2 & 3.7 & 21 & 38.9 & \\
\hline
\end{tabular}

\section{NA: Not applicable}


Table (7): Effect of the educational program on opinion among study and control groups at three phases: before, at first and second evaluations.

\begin{tabular}{|c|c|c|c|c|c|c|c|c|c|c|c|c|c|c|c|}
\hline \multirow{3}{*}{ Opinion } & \multicolumn{5}{|c|}{ Before program } & \multicolumn{5}{|c|}{ At First evaluation } & \multicolumn{5}{|c|}{ At Second evaluation } \\
\hline & \multicolumn{2}{|c|}{$\begin{array}{l}\text { Study } \\
\text { group } \\
(n=54)\end{array}$} & \multicolumn{2}{|c|}{$\begin{array}{c}\text { Control } \\
\text { group } \\
(n=54)\end{array}$} & \multirow{2}{*}{$\begin{array}{c}P \\
\text { value }\end{array}$} & \multicolumn{2}{|c|}{$\begin{array}{l}\text { Study } \\
\text { group } \\
(n=54)\end{array}$} & \multicolumn{2}{|c|}{$\begin{array}{c}\text { Control } \\
\text { group } \\
(n=54)\end{array}$} & \multirow{2}{*}{$\begin{array}{c}\mathbf{P} \\
\text { value }\end{array}$} & \multicolumn{2}{|c|}{$\begin{array}{l}\text { Study } \\
\text { group } \\
(n=54)\end{array}$} & \multicolumn{2}{|c|}{$\begin{array}{c}\text { Control } \\
\text { group } \\
(n=54)\end{array}$} & \multirow{2}{*}{$\begin{array}{c}\mathbf{P} \\
\text { value }\end{array}$} \\
\hline & & $\%$ & & $\%$ & & No. & & $\begin{array}{c}\text { No } \\
\text {. }\end{array}$ & $\%$ & & No. & $\%$ & & $\%$ & \\
\hline \multicolumn{16}{|c|}{ Patient belief about heart failure } \\
\hline Can be healed & 4 & 7.4 & & 33.3 & \multirow{8}{*}{$0.001^{*}$} & 1 & 1.9 & 18 & 33.3 & \multirow{8}{*}{$0.001^{*}$} & 0 & 0.0 & 18 & 33.3 & \multirow{8}{*}{$0.001 *$} \\
\hline $\begin{array}{l}\text { Disease disabling } \\
\text { patients completely }\end{array}$ & & 44.4 & & 0.0 & & 7 & 13.0 & 1 & 1.9 & & 3 & 5.6 & 1 & 1.9 & \\
\hline $\begin{array}{l}\text { Group of symptoms } \\
\text { can be controlled }\end{array}$ & 4 & 7.4 & & 13.0 & & 15 & 27.8 & 0 & 0.0 & & 10 & 18.5 & 0 & 0.0 & \\
\hline Stop heart beating & 10 & 18.5 & & 24.1 & & 0 & 0.0 & 10 & 18.5 & & 1 & 1.9 & 10 & 18.5 & \\
\hline $\begin{array}{l}\text { Week and irregular } \\
\text { heart beats }\end{array}$ & 6 & 11.1 & & 9.3 & & - & - & - & - & & - & - & - & - & \\
\hline $\begin{array}{l}\text { Weak heart leads to } \\
\text { edema }\end{array}$ & 0 & 0.0 & & 20.4 & & 0 & 0.0 & 11 & 20.4 & & 0 & 0.0 & 11 & 20.4 & \\
\hline All & 6 & 11.1 & & 0.0 & & - & - & - & - & & - & - & - & - & \\
\hline $\begin{array}{l}\text { Inability of heart to } \\
\text { pump enough blood }\end{array}$ & - & & & - & & 31 & 57.4 & 14 & 25.9 & & 40 & 74.1 & 14 & 25.9 & \\
\hline \multicolumn{16}{|c|}{ The most important factor affecting your disease } \\
\hline $\begin{array}{l}\text { Balanced programs of } \\
\text { rest and activity }\end{array}$ & 9 & 16.7 & 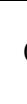 & 0.0 & \multirow{6}{*}{$0.001 *$} & 2 & 3.7 & 0 & 0.0 & \multirow{6}{*}{$0.001 *$} & 5 & 9.3 & 1 & 1.9 & \multirow{6}{*}{$0.001 *$} \\
\hline Psychic rest & 0 & 0.0 & & 51.9 & & 2 & 3.7 & 28 & 51.9 & & 0 & 0.0 & 28 & 51.9 & \\
\hline Drugs & 4 & 7.4 & & 0.0 & & - & - & - & - & & 2 & 3.7 & 0 & 0.0 & \\
\hline Nothing & 20 & 37.0 & & 18.5 & & 0 & 0.0 & 3 & 5.6 & & 2 & 3.7 & 0 & 0.0 & \\
\hline $\begin{array}{l}\text { All except weakness of } \\
\text { pulse rate }\end{array}$ & 19 & 35.2 & 1 & 29.6 & & 50 & 92.6 & 23 & 42.6 & & 0 & 0.0 & 3 & 5.6 & \\
\hline All of the above & 2 & 3.7 & & 0.0 & & - & - & - & - & & 45 & 83.3 & 22 & 40.7 & \\
\hline \multicolumn{16}{|c|}{ What are the difficulties in your opinion } \\
\hline Diabetes & 3 & 5.6 & 18 & 33.3 & \multirow{4}{*}{$0.001 *$} & 3 & 5.6 & 18 & 33.3 & \multirow{4}{*}{$0.001^{*}$} & 3 & 5.6 & 18 & 33.3 & \multirow{4}{*}{$0.001 *$} \\
\hline Kidney disease & 4 & 7.4 & 0 & 0.0 & & - & - & - & - & & - & - & - & - & \\
\hline Heart disease & 37 & 68.5 & 2 & 46.3 & & 44 & 81.5 & 26 & 48.1 & & 41 & 75.9 & 26 & 48.1 & \\
\hline All of the above & 10 & 18.5 & 1 & 20.4 & & 7 & 13.0 & 10 & 18.5 & & 10 & 18.5 & 10 & 18.5 & \\
\hline
\end{tabular}




\section{DISCUSSION:}

Congestive heart failure is a long-term illness that is characterized by multiple problems for patients and their families. Therefore, it is very important that patients know information to cope with their problems as following the prescribed therapeutic regimen which includes life style modifications related to diet, activity and medication (Lakdizaji et al., 2013). Recognition and treatment of problems are priorities of care for patients with chronic illness such as congestive heart failure (Richman, 2010).

Knopp (2009); Delaney et al. (2011) mentioned that nurses can provide assistance and information to patients and their families while in hospital by discussing with them some of the common problems which patients may experience once they return home. It is important as well for nurses to be aware of the feelings and needs of the spouse or carer. According to Thompson (2010), the importance of individualizing a plan of care before discharge should include home care for provision of long-term education and counseling.

The results of the present study revealed that age of most of the patients in both control and study groups was 60 years old and more. This result is supported by Karlsson (2011), who found that the congestive heart failure is more common in aged persons over 65 year. Moreover, American Heart Society (2012); Cardiology Channel (2012), found that congestive heart failure is more common in persons aged between 40 to 55 years old, while Morgan \& Naka (2013) reported that, age and gender are major factors in developing heart disease. Additionally, prevalence of CHF is increased in older people particularly after 45 years of age.

In relation to gender, it was found that the majority of the studied patients in both control and study groups were male patients. This fact may be explained by increase of smoking habit among men, in addition to occupational factor that need increased physical work. This result was explained by Lakdizaji et al. (2013) who found that during a study examined data from general practices of 2.1 million patients, age related prevalence of heart failure was 9.7 per 1.000 males and 8.2 per 1.000 females.

Regarding the marital status, it was found that majority of studied patients in both control and study groups were married, this finding goes in the line with Elsayed (2004); Reddy et al. (2010), who found that married patients represented the higher percentage compared to single, divorced and widow patients. So, stress of the family obligation could be a contributing factor to develop CHF.

In relation to educational level, the current study revealed that less than half of the subjects were able to read and write in each group. Additionally, the majority of the study subjects were laborers, with limited education. Similarly, Moore et al. (2014), reported that educational level is considered a personal issue which influences the individual ability to cope with the disease. While, Lakdizaji et al. (2013) reported that the level of education is important for CHF patients to perceive information. 
Regarding family income, more than half of the subjects in both control and study groups earned less than $300 \mathrm{LE}$ as monthly income. Also more than half them had insufficient income to cover the cost of treatment. However, less than half of the subjects were been supported by health insurance. Coyle \& Berger (2013), stated that lower socio-economic status and low income are associated with increased occurrence of chronic heart disease in both men and women probably due in part to the higher prevalence of CHF risk factors.

Regarding knowledge about history of disease, the majority of the subjects involved in both study and control group had incomplete knowledge about their own diagnosis, which includes definition, causes, types and complications of CHF. Such result in supported by Khan (2006); Gerdes \& Lorenz (2013) found that the majority of patients with CHF were concerned about causes and manifestations while only a minority were concerned about diagnosis, prevention and complications of CHF. While Colet et al. (2014), stated that, only a small portion of the study sample was able to correctly answer CHF knowledge questions that entailed prevention of the disease.

All the studied subjects in both groups suffered from chronic disease such as hypertension, diabetes, myocardial infarction, angina and/or anemia. Regarding number of comorbid diseases, it was found that the majority of the patients were hypertensive and diabetic while more than half of the patients had myocardial infarction. This finding is also congruent with what was reported by Henein (2010) who revealed that $\mathrm{CHF}$ results from excessive strain on the heart, as hypertension increases cardiac workload.

The present study showed that the majority of the subjects in both study and control groups suffered from gradual onset of shortness of breath that worsened in response to a variety of activities. Moreover, patients had moderate dyspnea in response to light activities (e.g., making a bed or walking across the room) Perez et al. (2014), found that, "effort" is the precipitating factor number one for dyspnea in CHF patients, followed by gradually progressive fatigue.

In relation to history of previous hospital admission, it was found that the majority of the subjects in the study and control group had history of previous hospital admissions. Less than half of patients were admitted to the hospital for two weeks each year. This may be due to non compliance with the treatment regimen. This result was supported by Pang (2011); Blecker et al., (2014), who stated that, improved pharmaceutical management, close follow-up, and patient education about self-management had resulted in reduced hospital admission. Also when the studied patients had telephone contact to medical team and were referred to the general practitioner for further help, heart failure hospitalization was reduced by $25 \%$ but did not reduce all causes of hospital admissions or mortality. 
Findings of the present study showed that the majority of the studied subjects in both study and control groups were suffering from fatigue. The patients described fatigue as an intermittent problem that affected their body and their ability to think. This result was supported by Moore et al. (2014); Perez et al. (2014) who conducted an interventional study on 542 patients with CHF in UK General Hospital. They reported that among participants who experienced fatigue daily, factors contributing to fatigue were identified as, being female, being staged according to New York Heart Association as class III or IV, having symptoms of depression and/or having two or more comorbidities. Interviews identified other symptoms, including chest pain, nausea, sleep disruption, and confusion. The authors added that nurses can help the patients to plan their activities so that they can rest before any anticipated outing.

The present study indicated that the majority of the subjects in both groups complained from dyspnea as the most common reason for which they sought medical care. Butler (2012), stated that dyspnea is the single most important factor contributing to functional difficulties in health clinics visits among CHF patients. Patients described their shortness of breath as "fullness that comes up through the neck".

The current study showed that the majority of the subjects in both groups were suffering from pain, especially chest pain, which affected their physical and psychological status. The patients reported that they are able to learn to differentiate types of chest pain, which was consistent with the results of Damjanov (2012) who found that, patients reported troubling sensations when lying on their left sides. They described sensations of hearing their heart beat and feeling it pounding. Most of the patients who reported these sensations were so problematic that they were unable to sleep on their left side.

The present study indicate the that majority of the subjects in the study and control group combined of sleep disturbance, mostly due to shortening of breath, chest pain and/or cough. This result is supported by Goldberg (2010), who reported that, the consequence of disturbed sleep could be daytime sleepiness, which can affect negatively all dimensions of quality of life. In response to difficult sleeping, patients used distracting thinking through reading, watching television, and drinking herbal tea. They took sedative and antidepressants to improve sleep. The study group had higher knowledge about how to regulate sleep and rest. The patients need longer time in bed and have the worst sleep quality.Such result is consistent with Eltahry (2008), who found that sleep affects all aspects of quality of life, including general health, physical functioning, cognitive functioning, daily activities, and work performance. Sleep disturbance has a profound effect on physical functioning, and psychological status in CHF patients.

The result of the present study showed that the majority of the subjects in the study and control patients illness had disturbed sexual life, which is in agreement with 
Polling (2013); Mark et al. (2014), who found that the patients with congestive heart failure had specific factors that enhance risk for sexual dysfunction, such as low cardiac output and use of drugs with vasodilator effect. Sexual dysfunction can negatively affect interpersonal relationship and self-esteem, with significant impact on the quality of life. The studied patients had sexual problems mainly in the from of dyspnea, fatigue and tachycardia which occur during sexual intercourse while others complained from sexual inability. This finding goes in line with Thompson (2012), who found that the majority of his/her study subjects had lost sexual activities or had sexual performance problems. These findings also were supported by McGee (2012) who stated that symptoms of CHF influence sexual performance. Sexual performance is not only influenced by shortness of breath and fatigue but also by anxiety accompanying physical activities.

The present study indicated that the study group subjects had significantly higher level of anxiety about the disease, lower sense of less affection on behavior and vision to life as compared to the control group at all timings of evaluation. A significantly higher percentage of the study group was afraid from disease complications compared to the control group before and immediately after the program. At second evaluation after the program, such difference disappeared between both groups as such fear decreased among the study group, which could be explained by better adaptation and acceptance for the disease. Moreover, patients gained a sense of power in controlling the situation through reassurance, encouragement, talking to a close person about their worries and avoiding over thinking about their own illness, These findings are consistent with those of Jenner et al. (2009); Lefteriotis (2013) who studied the strategies used by CHF patients to control psychological problems, they found that, using the following strategies: being optimistic and praying when upset, sense of security and privacy, accepting meanwhile strategy enjoyment was not reported at all.

The current study revealed a highly statistically significant difference between the control and study group regarding patients beliefs about heart failure. Among the study group before program the patients believed that it is a disabling disease that affects patients completely, mean while at the immediate, and post intervention assessments, the patients corrected their answers as inability of heart to pump enough blood which was among the study than control group. The patients had lack of information about the disease, factors affecting the disease, psychological issues, physical activity, work, rest/sleep, sexual life, treatment and health instructions. It is mandatory that patients receive appropriate information to meet their needs, right of self-determination and to make meaningful choices. This result was supported by Andrietta et al. (2011); Albert (2013), who found that the patients were capable of making decisions provided they have proper information. Prerequisite to get information and counseling is properly functioning communication between health care and patients. 
In conclusion, nurses play important role to meet patient's information needs through different methods. Health education should be accustomed according to patient's condition, level of education, occupation, and socioeconomic condition. Indeed there is a need for continuing care of the CHF patient's after discharge. This was supported by Karen (2009), who showed that the requirements of a good system of follow-up such as monitoring of patient symptoms, medication reviews, support in life style change and the provision of help if necessary. The patients must receive care and emotional support from their families. This reason comes in accordance with a study conducted by Clark et al. (2009), who stated that among the chronically ill, positive associations have been shown between information, life satisfaction and social contact.

\section{CONCLUSION:}

Based on study findings, it can be concluded that:

Educational program has a significant improvement on the patient's knowledge regarding congestive heart failure

\section{RECOMMENDATIONS:}

Patients and their families should be educated about CHF, weight controlling and regular weight checking, decrease heart effort, prevention of infectious disease, physical activity, deep respiration exercise, avoid carrying heavy objects, regular checking of medical investigations, disease affects work and rest periods between activity, stress management and early warning signs during sexual relation .Developing the patient's information through using patients education booklet, a HF video, and written information sheet that the patients may take home each time they are seen in the hospital or clinic, also incorporating color picture flash cords of commonly used heart failure medication into initial and follow up teaching to assist patients in identifying medications and dosages. Specialty nursing programs to prepare nurses in different specialties to ensure high quality of information and care provided to patient. Replication of the current study on a large probability sample from different geographical areas to achieve more generalized results.

\section{REFERENCES}

Abu Donia SH. (1977): Educational program for patients with heart failure. Thesis for the degree of PHD, Medical Surgical Nursing, Faculty of Nursing, Alexandria University.

Albert N. (2013): Parallel paths to improve heart failure outcomes. American Journal of Critical Care. Vol. 22, No. 4; P.p. 289-297.

American Heart Society (2012): Heart failure, risk factors, Http:// www.failure.org / doctorate / CRI / content / CRI - 2-4-3-what - is - risk factors. 
Amorim A., Miriam M. and Ribeiro T. (2013): Nursing diagnose and interventions for patients with congestive heart failure, Journal of the American College of Cardiology, 47(2); P.p. 7-380.

Andrietta M., Moreira R. and Barros A. (2011): Hospital discharge plan for patients with congestive heart failure. Rev. Latino-Am. Enfermagem, Vol 19, No. 6; P.p. 521445 .

Australian Institute of Health and Welfare. (2016): information and statistics to promote better health and wellbeing. http//www.aihw.gov.au/mal-health/health-status.

Blecker S., Agarwal S. and Chang P. (2014): Quality of Care for Heart Failure Patients Hospitalized for Any Cause. Journal of the American College of Cardiology, Vol. 63, No.; P.p. 132-130.

Butler J. (2012): An overview of chronic heart failure management. Nursing Practice, Vol. 108, No 14/15; P.p. 16-20.

Cardiology Channel (2012): Heart failure, clinical manifestation, Http:// www.cardiology channel.com / heart failure / clinical manifestation. html.

Clark A., Savard L. and Thompson D. (2009): Evidence for Heart Failure DiseaseManagement Programs, Journal of the American College of Cardiology. Vol. 54, No. 5; P.p. 397-401 .

Colet J., Rotellar J. and Vela E. (2014): Primary Care Program for Heart Failure. Rev Esp Cardiol. Vol .67, no 4; P.p. 283-293.

Coyle D. and Berger R. (2013): Cost-utility analysis of NT-pro BNP-guided multidisciplinary care in chronic heart failure. International Journal of Technology Assessment in Health Care, VOL. 3, NO 11; P.p1-29.

Damjanov I. (2012): Pathology for the heart professions. $4^{\text {th }}$ edition. ElsevierSaunders.

Delaney C., Apostolidis B. and Lachapelle L. (2011): Home care nurses' knowledge of evidence-based education topics for management of heart failure. Heart \& Lung journal, vol. 40; P.p. 285-292.

Elsayed ES. (2004): Orientation guideline for patient undergoing cardiothoracic surgery. Thesis for degree of Msc., Medical Surgical Nursing, Faculty of Nursing, Ain Shams University; p.p. 60-83.

Eltahry SH. (2008): Assessment of Informational Needs of Patients with Congestive Heart Failure . Unpublished Master Degree. Faculty of Nursing - Suez Canal University (Medical Surgical Nursing). 
Gerdes P. and Lorenz R. (2013): The Effect of an Outpatient Interdisciplinary. Heart Failure Education Program. The Journal for Nurse Practitioners- JNP, Vol .9, no7; P.p. 422- 427.

Goldberg L. (2010): Heart Failure in the clinic. American College of Physicians, Annals of Internal Medicine; Vol, 44 P.p. 1-16.

Henein M. (2010): Heart Failure in Clinical Practices. Springer-Verlag London; P.p. 255-276.

Jenner R., Strodl E. and Schweitzer R. (2009): Anger and Depression Predict Hospital Use among Chronic Heart Failure Patients, Australian Health Review, Vol .33, No 4; P.p. 541-548.

Jensen SH. (2015): Nursing health assessment a best practice approach. $2^{\text {nd }}$ edition. Wolters Kluwer. P.p., 413-437.

Karen Y. (2009): The effect of shared medical visits on knowledge and self-care in patients with heart failure, Heart and Lung, (38): one; P.p. 25-33.

Karlsson R., Edner M. and Fvenmark C. (2011): professional education program for family members of patients with chronic heart failure. Patient Education and Counseling, vol, 85; P.p. 162-168.

Katz D., Friedman R. and Lucan S. (2015): Nutrition in clinical practice. $3^{\text {rd }}$ edition . Wolters Kluwer. P.p. 204-214.

Khan G. (2006): Approach practical: heart disease diagnosis and therapy. $3^{\text {rd }}$ ed., MCG Grow-hill Book company USA; p.p. 340-352.

Knopp A. (2009): Nurses Knowledge of Heart Failure Guidelines in A Western Montana Hospital. Master thesis, faculty of nursing, Montana State University; P.p.2035 .

Lakdizaji S., Hassankhni H. and Agdam A. (2013): Effect of Educational Program on Quality of Life of Patients with Heart Failure: A Randomized Clinical Trial. Journal of Caring Sciences, VOL. 2, NO 1; P.p 11-18.

Lefteriotis $\boldsymbol{C H}$. (2013): Depression in Heart Failure patients, Health science Journal, vol. 7, No 4; P.p. 349-355.

Mark H., Paris L. and Taylor L. (2014): A nurse-guided patient-centered heartcentered heart failure education program. Journal of Nursing Education and Practice, Vol. 4, No. 3; P.p. 49-57.

McGee S. (2012):-Evidence-based physical diagnosis .Philadelphia -Elsevier 
McMurray, JJ., Adamopoulos S., Anker SD. And Bohm M. (2012): ESC Guidelines for the diagnosis and treatment of acute and chronic heart failure. Eur J Heart Fail, 14(8), 803-69.

Moore L., Dalley F. and Agur A. (2014): Clinically oriented anatomy, $7^{\text {th }}$ edition, Lippincott Williams and Wilkins.

Morgan J. and Naka Y. (2013): Surgical Treatment for Advanced Heart Failure. Springer New York Heidelberg Dordrecht London; P.p.1-24.

Moser, DK., Dickson, Jaarsma T. and Lee C. (2012): Role of self-care in the patient with heart failure .Curr Cardiol Rep, 14(3), 265-75.

Pang P. (2011): Acute Heart Failure Syndromes Initial Management. Emerg Med Clin N Am 29; P.p. $675-688$

Perez A., Jhund P. and Macdonald M. (2014): Fatigue as a Predictor of Outcome in Patients with Heart Failure. American College of Cardiology Foundation, Vol. 2, No. 2; P.p. 188- 197.

Pinkerman C., Sander P., Breeding JE., Brink D., Curtis R. and Hayes R. (2013): Heart Failure in Adults. Institute for Clinical Systems Improvement, $13^{\text {th }}$ Edition; P.p. $9-15$

Polling G. (2013): Nurses rated highest in honesty and ethical standards. Retrieved from http//www.Gallup.com. Heart failure. British Columbia in Ministry of Health Services. Website: www.health.gov.bc.ca/msp

Reddy S., Bahl A. and Talwar K. (2010): How do we improve diagnosis \& management? Indian J Med Res. Vol .132, P.p. 549-560.

Richman H. (2010): Heart failure touching deep with music, emotional and physical; Http://www.sounfeetings.com/products/alternative-medicine / music - therapy / heart failure.

Thompson J. (2012): Cardiovascular problems. Philadelphia -Elsevier.

Thompson T. (2010): A Public Health Action Plan to Prevent Heart Disease and Stroke, American Heart Association; P.p.14-15.

Urden I., Stacy K. and Lough M. (2014): Critical care nursing ,diagnosis and management. $7^{\text {th }}$ edition, Elsevier Mosby. P.p. 366-374.

Walker B., Colledge N., Ralston S. and Penman I. (2014): Davidson's principles \& practice of medicine. $22^{\text {th }}$ edition, Churchill Livingstone Elsevier. P.p. 546-553.

Weber R. and Kelley H. (2014): Health assessment in nursing. $5^{\text {th }}$ edition, Lippincott Williams and Wilkins. P.p. 416-446. 
Williams I. and Hopper P. (2015): Understanding medical surgical nursing; $5^{\text {th }}$ edition .Davis Company ,Philadelphia. P.p. 522-544.

Zile M., Gottdiener J. and Hetzel S. (2011): Prevalence and significance of alterations in cardiac structure and function in patients with failure and a preserved ejection fraction . Circulation; vol , 124; P.p. 2491-501. 
تـأثـير البـرنـامــج التـعليمي لـمرضـي هبوط القلب وأثـره علي الــالـة الصـيـة

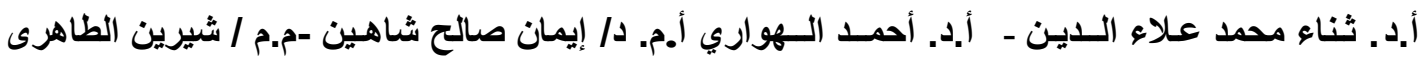

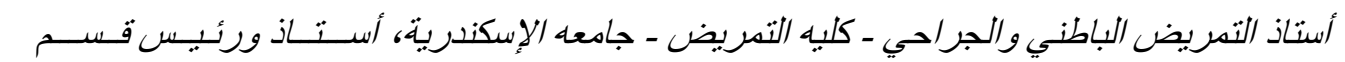

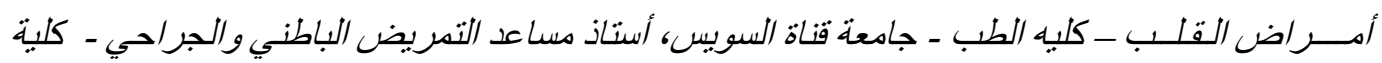

التمريض - جامعة بورسعبد، مدرس مساعد التصريض الباطني والجر/حي ـ كلية التمريض- جامعة بورسعيد

\section{الخلاصة}

الدراسة الحالية دراسة شبة تجريبية هدفها تقييم تأثثر البرنامج التعليمي علي معلومات مرضى هبوط القلب. وشملت عينة البحث (108) مريض بمستشفى بورسعيد العام و النصر و التضامن، وقد تم تجميع البيانات عن طريق استخدام استمارة استبيان للمرضى لتقييم معلوماتهم النظرية عن مرض هبوط القلب وتم تجميع الاستمار ات قبل البدء في البرنامج وبعد تتفيذ البرنامج مباثرة ثم بعد ثلاث شهور. وقد أسفرت نتائج البحث علي وجود تحسن واضح في معلومات المرضى بعد تتفيذ البرنامج مباشرة. بناء على نتائج الدر اسة أوصى بأن يتم عمل در اسة لتقييم الحالة الوظيفية لمرضي هبوط القلب أثناء دخولهم المستشفى و أثناء خروجهم لمعرفة التغيير في حالتهم الوظيفية تطبيق هذه الدر اسة علي نطاق عينة أكبر لتحقيق التعميم علي مدي أوسع في العلاج و الرعاية الصحيه ـ

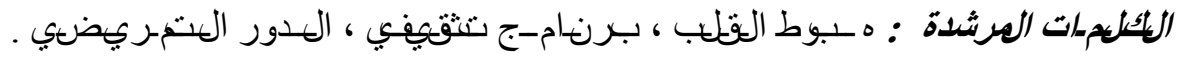

